\title{
Why do we need alternative tools to control mosquito-borne diseases in Latin America?
}

\author{
Rafael Maciel-de-Freitas $1 /{ }^{+}$, Raquel Aguiar ${ }^{2}$, Rafaela V Bruno ${ }^{3}$, \\ Maria Cristina Guimarães ${ }^{4}$, Ricardo Lourenço-de-Oliveira ${ }^{1}$, Marcos HF Sorgine ${ }^{5}$, \\ Cláudio J Struchiner6, Denise Valle', Scott L O'Neill ${ }^{6}$, Luciano A Moreira9 ${ }^{9}$
}

\author{
${ }^{1}$ Laboratório de Transmissores de Hematozoários ${ }^{2}$ Serviço de Jornalismo e Comunicação ${ }^{3}$ Laboratório de Biologia Molecular de Insetos \\ ${ }^{7}$ Laboratório de Fisiologia e Controle de Artropodes Vetores, Instituto Oswaldo Cruz ${ }^{4}$ Instituto de Comunicação e \\ Informação Cientifica e Tecnologica ${ }^{6}$ Programa de Computação Cientifica, Fiocruz, Rio de Janeiro, RJ, Brasil \\ ${ }^{5}$ Departamento de Bioquimica Médica, Universidade Federal do Rio de Janeiro, Rio de Janeiro, RJ, Brasil \\ ${ }^{8}$ School of Biological Science, University of Monash, Melbourne, Australia ${ }^{9}$ Laboratório de Malária, \\ Centro de Pesquisas René Rachou-Fiocruz, Belo Horizonte, MG, Brasil
}

In this opinion paper, we discuss the potential and challenges of using the symbiont Wolbachia to block mosquito transmitted diseases such as dengue, malaria and chikungunya in Latin America.

Key words: dengue - malaria - chikungunya - yellow fever - control - surveillance - Aedes aegypti

Among the diseases transmitted by mosquito vectors, malaria and arboviruses such as dengue, yellow fever, West Nile and chikungunya, are of great public health concern due to the occurrence of disease outbreaks, the collapse of overburdened health systems during such epidemics and the high morbidity and mortality rates registered in endemic regions. According to the Pan American Health Organization, the number of notified cases and deaths from dengue and malaria in Latin America is alarming. For instance, between 20012011, Latin America reported 9,059,896 dengue cases with 3,653 deaths, with Brazil accounting for $63.9 \%$ of the cases and $58.8 \%$ of deaths. A similar scenario has been observed for malaria between 2001-2010, with more than 8.2 million cases and 2,077 deaths in Latin America, with $50 \%$ of both cases and deaths being reported in Brazil (new.paho.org/index.php).

Historically, the most frequently used strategy to minimize mosquito-borne disease burden is the intensification of vector control efforts in areas with high transmission risk or disease incidence levels. However, in the case of dengue, the predictive identification of infestation/transmission hot spots in sufficient time for effective intervention is hampered by the poor correlation between the currently used larval abundance indices and the real density of adult mosquito vector populations. Similarly, direct surveillance of adult mosquito popula-

Financial support: MS/SCTIE)/CNPq, Monash University, NIH/ Global Health Initiative of the Bill \& Melinda Gates Foundation + Corresponding author: freitas@ioc.fiocruz.br

Received 10 August 2012

Accepted 4 September 2012 tion densities can be distorted if traps with low or biased capture efficiencies are used. Furthermore, as in the case of Rio de Janeiro (RJ), entry of health workers into some neighbourhoods is inadvisable due to high levels of urban violence, preventing the acquisition of entomological data necessary for implementing control strategies.

Since the beginning of the 20th century, mechanical control of the yellow fever and dengue vector Aedes aegypti has been based on removing container breeding sites, which is logistically challenging today given the size of many dengue endemic metropolitan areas. Similarly, mechanical control is frequently unfeasible for large river and lake margins, which are the breeding sites for Anopheles darling, the main malaria vector in Latin America. The advent of potent long-lasting chemical insecticides during the decade of 1940 was promising, but overdependence on this tool for vector control resulted in the evolution of resistance in natural populations, hindering its effectiveness in the long-term. Today, dissemination of insecticide resistance throughout vector populations is much faster than the rate of development of new insecticides. In addition, the existence of cross-resistance, based on the activation of general detoxifying mechanisms in the vector, can shorten the lifespan of alternative insecticides or even prevent their implementation. Finally, biological control is frequently cited as an environmentally-acceptable tool, but the production, maintenance and distribution of speciesspecific predators/parasitoids on a large-scale is often considered unfeasible.

Given that traditional control methods have shown limited success - primarily because of modern urban complexity, increasing insecticide resistance and the labour-intensive and logistically-demanding nature of mechanical control and entomological surveys, with their limited ability to accurately predict future epidemics - the development of new strategies for vector control 
must be encouraged. Currently, several different approaches have been envisaged, such as the use transgenic mosquitoes or adult mosquitoes as vehicles of juvenile hormone transfer between resting and oviposition sites, as well as studies utilizing the mosquito microbiota or its symbionts to manipulate disease transmission.

Indeed, a recent approach may be helpful in diminishing the incidence rates of some vector-borne diseases in human populations. The cornerstone of this strategy is to spread into natural mosquito vector populations an endosymbiont named Wolbachia, which is a maternallyinherited intracellular bacterium that is present in about $60 \%$ of different insects species worldwide, including other mosquitoes, butterflies, beetles, ants and bees. Curiously, Anopheles and Ae. aegypti mosquitoes are not naturally-infected with this bacterium, but natural infections are widespread in another dengue vector Aedes albopictus - and the common fruit fly Drosophila melanogaster. Interestingly, after introducing a Wolbachia strain from D. melanogaster into Ae. aegypti, it was observed that females of this mosquito carrying Wolbachia blocked dengue virus and significantly reduced the load of chikungunya and Plasmodium gallinaceum, an etiologic agent of avian malaria (Moreira et al. 2009). Other reports in Australia subsequently showed that mosquitoes carrying Wolbachia were able to invade wild populations of mosquitoes that do not carry the bacteria (Hoffmann et al. 2011). This happens because Wolbachia is able to manipulate host reproduction in such a way that infected individuals have higher chances of producing offspring, which will also carry the bacterium. These findings encouraged the establishment of the Eliminate Dengue Program (EDP), a global initiative to eliminate dengue transmission by the release of Ae. aegypti carrying Wolbachia (eliminatedengue.org). Simultaneously underway in Australia, Indonesia, Vietnam and China, the EDP is based on a multidisciplinary team including entomologists, mathematical modellers and community engagement and communication experts.

Several Brazilian scientists are now part of the EDP, an association between Fundação Oswaldo Cruz, the Brazilian Ministry of Health/Secretariat of Science, Technology and Strategic Inputs and Monash University from Australia, in an effort to reduce or even eliminate dengue transmission in some localities of RJ during the next five years. In order to evaluate if the Wolbachia strategy will be reliable for dengue control, a number of questions and challenges are first being investigated by the Brazilian EDP team, such as: (i) can Wolbachia block local dengue serotypes and genotypes in Brazilian Ae. aegypti mosquitoes, (ii) is it possible to evaluate biological aspects of natural Ae. aegypti populations such as dispersal, daily survival probability, fecundity and biting rates before, during and after releases of Wolbachiainfected mosquitoes, (iii) can Wolbachia be introduced into insecticide susceptible Ae. aegypti, which can then be released into the field, enabling a reduction in insecticide resistance levels of natural Ae. aegypti populations, (iv) can the number, periodicity, seasonality and sites of mosquito releases be modelled, as well as their effectiveness when used in association with other control measures, such as a dengue vaccine, (v) are there changes in Ae. aegypti gene expression, immune response, circadian rhythm and insecticide resistance profile due to Wolbachia and (vi) can community engagement and support for release of Wolbachia-infected mosquitoes be obtained, together with a plan to prior reduce local mosquito population to ensure that community safety concerns are allayed.

Historical evidence has shown that any control method should not be seen as a silver bullet, but as part of a broader integrated management vector control program. Under this perspective, it is peremptory to say the $\mathrm{Wol}-$ bachia strategy alone will control disease transmission, even though it has shown promising results under laboratory, semi-field and field conditions up to now. Thus, it may result in significant decrease in the incidence rates of some diseases transmitted by mosquitoes, if used together with appropriate mechanical and chemical interventions and continuous community participation. Furthermore, this approach is an environmentally friendly, sustainable and a not-for-profit initiative that can be complementary to the strategies currently used by the Ministry of Health, as well as used together with a dengue vaccine, that is presently being tested, to potentially eliminate dengue transmission.

\section{REFERENCES}

Hoffmann AA, Montgomery BL, Popovici J, Iturbe-Ormaetxe I, Johnson PH, Muzzi F, Greenfield M, Durkan M, Leong YS, Dong Y, Cook H, Axford J, Callahan AG, Kenny N, Omodei C, McGraw EA, Ryan PA, Ritchie SA, Turelli M, O’Neill SL 2011. Successful establishment of Wolbachia in Aedes populations to suppress dengue transmission. Nature 476: 454-457.

Moreira LA, Iturbe-Ormaetxe I, Jeffery JA, Lu G, Pyke AT, Hedges LM, Rocha BC, Hall-Mendelin S, Day A, Riegler M, Hugo LE, Johnson KN, Kay BH, McGraw EA, van den Hurk AF, Ryan PA, O'Neill SL 2009. A Wolbachia symbiont in Aedes aegypti limits infection with dengue, chikungunya and Plasmodium. Cell 139: 1268-1278. 